\title{
Silencing Genes Encoding Omega-3 Fatty Acid Desaturase Alters Seed Size and Accumulation of Bean pod mottle virus in Soybean
}

\author{
Ajay Kumar Singh, ${ }^{1}$ Da-Qi Fu, ${ }^{1}$ Mohamed El-Habbak, ${ }^{1}$ Duroy Navarre, ${ }^{2}$ Said Ghabrial, ${ }^{1}$ and \\ Aardra Kachroo ${ }^{1}$ \\ ${ }^{1}$ Department of Plant Pathology, University of Kentucky, Lexington 40546 U.S.A.; ${ }^{2}$ Department of Plant Pathology, United \\ States Department of Agriculture-Agricultural Research Service, Washington State University, Prosser 99350, U.S.A.
}

Submitted 8 September 2010. Accepted 24 November 2010.

\begin{abstract}
Omega-3 fatty acid desaturase (FAD3)-catalyzed conversion of linoleic acid to linolenic acid (18:3) is an important step for the biosynthesis of fatty acids as well as the phytohormone jasmonic acid (JA) in plants. We report that silencing three microsomal isoforms of GmFAD3 enhanced the accumulation of Bean pod mottle virus (BPMV) in soybean. The GmFAD3-silenced plants also accumulated higher levels of JA, even though they contained slightly reduced levels of 18:3. Consequently, the GmFAD3-silenced plants expressed JA-responsive pathogenesis-related genes constitutively and exhibited enhanced susceptibility to virulent Pseudomonas syringae. Increased accumulation of BPMV in GmFAD3-silenced plants was likely associated with their JA levels, because exogenous JA application also increased BPMV accumulation. The JA-derived increase in BPMV levels was likely not due to repression of salicylic acid (SA)-derived signaling because the GmFAD3-silenced plants were enhanced in SA-dependent defenses. Furthermore, neither exogenous SA application nor silencing the SA-synthesizing phenylalanine ammonia lyase gene altered BPMV levels in soybean. In addition to the altered defense responses, the $G m F A D 3$-silenced plants also produced significantly larger and heavier seed. Our results indicate that loss of GmFAD3 enhances JA accumulation and, thereby, susceptibility to BPMV in soybean.
\end{abstract}

Soybean (Glycine max (L.) Merr.), the second largest crop produced in the United States, is an important source of protein and oil for both humans and animals worldwide. The obvious economic value of this crop underscores the importance of developing an in-depth understanding of the molecular mechanisms governing soybean development and stress responses. The availability of the soybean genome sequence in conjunction with a large number of molecular resources and genomic tools has greatly facilitated improved efforts toward understanding various aspects of soybean physiology. One such important tool includes the Bean pod mottle virus (BPMV)-based

Corresponding author: A. Kachroo; Telephone: (859) 257 7445; Fax: (859) 323 1961; E-mail: apkach2@uky.edu

Current address of D.-Q. Fu: College of Food Science \& Nutritional Engineering, China Agricultural University, Beijing, China.

* The $\boldsymbol{e}$-Xtra logo stands for "electronic extra" and indicates that six supplementary figures and one supplementary table are published online and that Figures 1 and 6 appear in color online. vector that can be used for both heterologous gene expression and silencing endogenous sequences in soybean (Zhang and Ghabrial 2006). BPMV, a member of the genus Comovirus in the family Comoviridae (Lomonossoff and Ghabrial 2001), has a bipartite positive-strand RNA genome consisting of RNA1 (approximately $6.0 \mathrm{~kb}$ ) and RNA2 (approximately 3.6 $\mathrm{kb})$ that are separately encapsidated in isometric particles. The BPMV genome is expressed via the synthesis and subsequent proteolytic processing of polyprotein precursors. The BPMVbased soybean expression vector is derived from RNA2 of the virus, which codes for a putative cell-to-cell movement protein (MP) and two coat proteins (CPs) (large [L] and small [S]). BPMV RNA2 was modified to enable the insertion of target sequences (between regions encoding the MP and L-CP). To ensure proper processing of the recombinant RNA2, additional proteinase cleavage sites flanking the sites of target sequence insertion were generated. Because the BPMV RNA2 vector alone is not infectious, transcripts from BPMV RNA1 are included in all inoculations with empty or recombinant RNA2 vector. The efficacy of this vector for use in soybean was first demonstrated both by expressing the green florescent protein (GFP) and by silencing the endogenous phytoene desaturase gene to produce a photobleaching phenotype in soybean (Zhang and Ghabrial 2006). We have recently used this vector to study soybean defense responses to a variety of microbial pathogens (Fu et al. 2009; Kachroo et al. 2008; Selote and Kachroo 2010).

Manipulating seed-related traits is an important aspect of improving oil crops such as soybean. Thus, it would be highly desirable to be able to apply functional analysis tools such as the BPMV-based vector to study or manipulate seed traits in soybean. However, BPMV is not known to be seed transmitted (Giesler et al. 2002; Skotland 1958). This is consistent with our report that the BPMV-based vector could not be detected in germinating embryos obtained from BPMV-infected plants (Kachroo et al. 2008). Furthermore, use of this vector for silencing endogenous gene expression has shown that the target genes are effectively silenced in the leaf, stem, flower, and root tissues but not in germinating embryos from infected soybean plants. For example, silencing the stearoyl-acyl carrier proteindesaturase genes (GmSACPDs-encoded proteins are involved in desaturation of the fatty acid [FA] stearic acid to oleic acid) in soybean resulted in the alteration of their FA profile in the vegetative and reproductive tissues but not in seed (Kachroo et al. 2008). However, seed obtained from the GmSACPD-silenced plants were severely altered in morphology. This suggested that altered gene expression in nonseed tissues could affect 
seed-related traits. Here, we investigated the efficacy of using the BPMV-based vector to alter seed traits by targeting genes that contribute to the synthesis of polyunsaturated FAs in seed oil. The high polyunsaturated FA content of normal soybean oil results in low oxidative stability, which can generate rancidity and reduce the shelf-life of food products as well as increase the viscosity of soy-based biodiesels (Canakci et al. 1999). Toward addressing this issue, breeding efforts have resulted in the generation of a number of cultivars containing oil with low levels of the polyunsaturated FA, linolenic acid (18:3) (Fehr et al. 1992; Hammond and Fehr 1983; Wilcox and Cavins 1985). Specifically, mutations in or RNAi-mediated downregulation of the omega-3 FA desaturase (FAD3) gene, whose gene product (FA desaturase 3) incorporates a double bond in linoleic acid (18:2) to produce 18:3, have been shown to lower 18:3 content in soybean oil (Chappell and Bilyeu 2006; Flores et al. 2008; Reinprecht et al. 2009). At least three FAD3 genes (designated $G m F A D 3 A-C$ ) have been identified in soybean, with $G m F A D 3 A$ showing the highest expression during embryogenesis and likely being the major contributor of 18:3 content in soybean oil (Bilyeu et al. 2003). We show that silencing three microsomal isoforms of FAD3 genes ( GmFAD3A, $G m F A D 3 B$, and $G m F A D 3 C$ ) of soybean using the BPMV-based vector alters 18:3 levels in the plant. Surprisingly, the GmFAD3silenced plants produced seed that were significantly larger in size, resulting in increased seed yield. Strikingly, the GmFAD3silenced plants also exhibited marked increase in the accumulation of BPMV, which was associated with increased levels of jasmonic acid (JA) in these plants.

\section{RESULTS}

Silencing the GmFAD3 genes alters the FA profile of soybean plants.

A recombinant vector targeting all three GmFAD3 genes was generated using a 372-bp fragment (nucleotide positions 424 to 795) from GmFAD3A. This region is highly conserved among all three isoforms (Supplementary Fig. 1). Soybean plants (cv. Essex) were inoculated with the empty BPMV vector or the recombinant vector targeting GmFAD3A, GmFAD $3 B$, and $G m F A D 3 C$. Reverse-transcription polymerase chain reaction (RT-PCR) analysis detected the expression of all three GmFAD3 genes in the root, stem, leaf, and petiole of vectorinfected plants (Fig. 1A). In contrast, the GmFAD3-silenced plants showed markedly reduced expression of GmFAD $3 A$, $G m F A D 3 B$, and $G m F A D 3 C$ in the leaf, petiole, stem, and root of GmFAD3-silenced plants, indicating that the GmFAD3 genes were successfully silenced in the vegetative tissues of soybean (Fig. 1A).

Because the FAD3 protein desaturates 18:2 to 18:3 in plants, we first analyzed the GmFAD3-silenced plants for their FA profiles. As reported earlier, infection with BPMV did not significantly alter FA levels in soybean plants (Kachroo et al. 2008). In comparison, leaf tissue from GmFAD3-silenced plants showed approximately 1.5 -fold increase in the percent mole levels of 18:2 and approximately 1.2-fold reduction in 18:3 compared with vector-infected plants (Table 1). Likewise, the absolute levels (milligram per gram of fresh weight) of these FAs were also altered in the leaf tissues of GmFAD3-
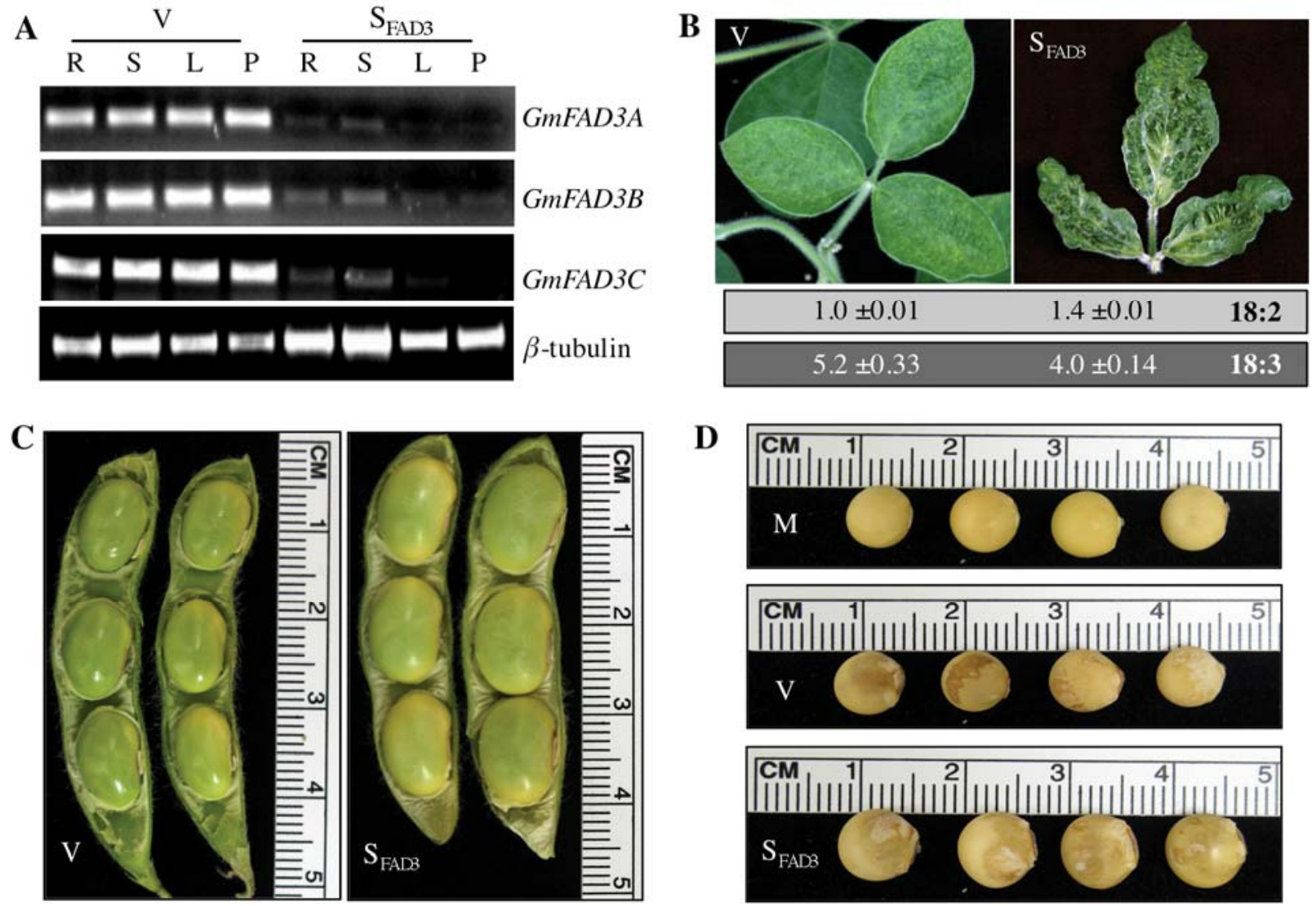

D
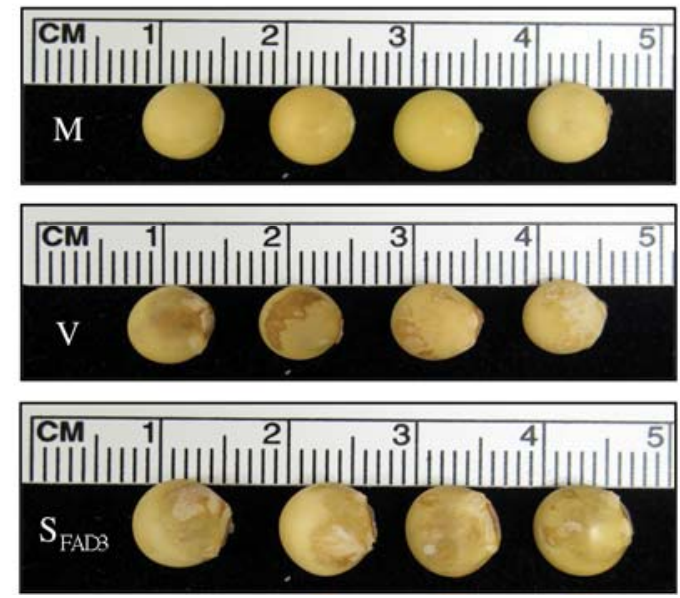

Fig. 1. Silencing Glycine max omega-3 fatty acid desaturase (GmFAD3) genes alters seed size in soybean. A, Reverse-transcription polymerase chain reaction analysis showing expression of the GmFAD3 isoforms in the root (R), stem (S), petiole (P), and leaf (L) tissue from vector-infected (V) and GmFAD3silenced $\left(\mathrm{S}_{\mathrm{FAD} 3}\right)$ plants. $\beta$-Tubulin levels were used as internal control for cDNA amounts. B, Morphological phenotype of leaves from V or $\mathrm{S}_{\mathrm{FAD} 3}$ plants. Numbers in lower panels indicate levels (milligrams per gram of fresh weight) of 18:2 and 18:3 in leaf tissues of respective plants; \pm indicates standard deviation of the mean $(n=3)$. C, Morphology and size of immature seed from V and $\mathrm{S}_{\mathrm{FAD} 3}$ plants. D, Morphology and size of mature seed from mock-inoculated $(\mathrm{M}), \mathrm{V}$, and $\mathrm{S}_{\mathrm{FAD} 3}$ plants. 
silenced plants (Fig. 1B, lower panels). Silencing of GmFAD3 also altered 18:2 and 18:3 levels in the petiole tissues; however, these alterations were less prominent in stem and root tissues. Furthermore, increase in 18:2 and decrease in 18:3 levels was also observed in the immature but not mature seed from GmFAD3-silenced plants (Table 1).

Silencing the GmFAD3 genes enhances soybean seed yield.

Although the GmFAD3-silenced plants exhibited severely enhanced BPMV-related symptoms (Fig. 1B; Supplementary Fig. 2), they produced pods with seed that were larger in size than those from vector-infected plants (Fig. 1C). Comparison of seed size and weight in greenhouse-grown vector-infected and GmFAD3-silenced plants (cv. Essex) showed that both immature and mature seed from GmFAD3-silenced plants were larger than those from mock-inoculated or vector-infected plants; seed from GmFAD3-silenced plants were approximately $19 \%$ wider and approximately $15 \%$ longer than those from vector-infected plants (Figs. 1C and D and 2A). Seed from GmFAD3-silenced plants also weighed more than those from mock-treated or vector-infected plants, with an approximately 55\% increase in seed weight per 100 seeds (Fig. 2B). We further tested the effect of silencing GmFAD3 on seed weight in other cultivars. Seed obtained from GmFAD3-silenced plants of cvs. Harosoy, Harosoy 63, Clark, and Merit all showed significant increases in weight compared with seed from vector-infected plants of the corresponding cultivars (Supplementary Fig. 3). To test whether the increase in seed size and weight was compensated for by a decrease in seed number, we compared seed number as well as the number of pods from mock-treated, vector-infected, and GmFAD3-silenced plants. No significant changes in total seed number were observed among mock-treated, vector-infected, and GmFAD3-silenced plants, indicating that neither BPMV infection nor silencing GmFAD3 altered seed number (Fig. 2C). Interestingly, both vector-infected and GmFAD3-silenced plants produced significantly reduced numbers of three-seeded pods per plant compared with mock-treated plants $(P$ value $<0.001$, per Student's $t$ test, $n=8$ ), suggesting that BPMV infection affected seed production per pod. However, these plants produced correspondingly higher numbers of one-seeded pods per plant, so that the total number of seed from vector-infected or GmFAD3-silenced plants did not differ significantly $(P=0.38$ and 0.098 for vector-infected and GmFAD3-silenced, respectively) from mock-treated plants (Table 2; Fig. 2C). This indicated that the increase in seed weight was not compensated for by a reduction in seed number in the GmFAD3-silenced plants. To determine whether seed from GmFAD3-silenced plants were altered in other seed attributes, we analyzed their protein, oil, and fiber contents. No changes were observed in any of these attributes (Supplementary Table 1). Our results show that silencing $G m F A D 3$ genes in soybean increases seed yield without affecting seed protein or oil content in greenhouse-grown plants. However, similar to seed from vector-infected plants, seed from GmFAD3-silenced plants exhibited mottling of the seed coat (Fig. 1D).

\section{GmFAD3-silenced plants accumulate increased levels of BPMV.}

We next compared BPMV levels in the GmFAD3-silenced and vector-infected plants (cv. Essex) plants, because these plants exhibited enhanced BPMV-related phenotypes (Fig. 1B). BPMV levels were monitored in the second and third emerging trifoliolates using BPMV CP-specific antibodies in an enzyme-linked immunosorbent assay (ELISA). Compared with vector-infected plants, the GmFAD3-silenced plants contained approximately four- and ninefold higher levels of BPMV in their second and third trifoliolates, respectively (Fig. $3 \mathrm{~A})$. Increased accumulation of BPMV in the GmFAD3silenced plants was confirmed using Western blot analysis. Increased amounts of both L-CP and S-CP of BPMV were detected in the leaf extracts of GmFAD3-silenced plants compared with those from vector-infected plants (Fig. 3B). These results showed that silencing GmFAD3 genes enhanced BPMV accumulation in soybean.

\section{GmFAD3-silenced plants accumulate increased levels of JA.}

To test whether the increased accumulation of BPMV in the GmFAD3-silenced plants was associated with changes in defense-related physiologies, we first tested the levels of the phytohormone JA in the GmFAD3-silenced plants. This is because the GmFAD3-silenced plants contain slightly lower levels of the FA 18:3, which is a precursor of JA biosynthesis. Analysis of JA levels showed that vector-infected plants accumulated nearly

Table 2. Pod number from control and GmFAD3-silenced plants

\begin{tabular}{lccc}
\hline & \multicolumn{3}{c}{ Number of pods $^{\mathbf{a}}$} \\
\cline { 2 - 4 } Plant & 1 seed/pod & 2 seeds/pod & 3 seeds/pod \\
\hline Mock-treated & $31 \pm 6.6$ & $69 \pm 5.7$ & $13 \pm 4.03$ \\
Vector-infected $^{\text {FAD3-silenced }}{ }^{\mathrm{b}}$ & $52 \pm 7.9$ & $69 \pm 6.3$ & $6 \pm 1.6$ \\
\hline
\end{tabular}

a Amount \pm standard deviation $(n=8)$.

b Plants silenced for omega-3 fatty acid desaturase (FAD3) gene GmFAD3.

Table 1. Altered fatty acid profile of GmFAD3-silenced plants

\begin{tabular}{|c|c|c|c|c|c|c|}
\hline \multirow[b]{2}{*}{ Plant, tissue } & \multicolumn{6}{|c|}{ Fatty acid $(\mathrm{mol} \%)^{\mathrm{a}}$} \\
\hline & $16: 0$ & 16:1 & 18:0 & $18: 1$ & 18:2 & $18: 3$ \\
\hline \multicolumn{7}{|l|}{ Vector-infected } \\
\hline Root & $27.7 \pm 1.0$ & $0.1 \pm 0.1$ & $5.6 \pm 0.2$ & $4.2 \pm 0.7$ & $41.0 \pm 3.0$ & $21.3 \pm 4.6$ \\
\hline Stem & $24.3 \pm 0.2$ & $0.3 \pm 0.1$ & $5.9 \pm 0.4$ & $4.4 \pm 0.3$ & $31.4 \pm 0.1$ & $33.5 \pm 0.5$ \\
\hline Leaf & $18.0 \pm 0.7$ & $3.9 \pm 0.1$ & $4.1 \pm 0.4$ & $2.3 \pm 0.1$ & $16.7 \pm 1.1$ & $54.9 \pm 1.9$ \\
\hline Petiole & $22.5 \pm 0.3$ & $0.06 \pm 0.0$ & $6.7 \pm 0.1$ & $2.6 \pm 0.1$ & $32.7 \pm 0.3$ & $35.3 \pm 0.7$ \\
\hline Immature seed & $11.3 \pm 0.8$ & $0.03 \pm 0.1$ & $4.2 \pm 0.2$ & $8.2 \pm 0.5$ & $29.1 \pm 0.9$ & $30.7 \pm 1.7$ \\
\hline Mature seed & $16.3 \pm 0.7$ & NA & $2.9 \pm 0.1$ & $13.4 \pm 0.7$ & $57.9 \pm 0.3$ & $9.5 \pm 0.2$ \\
\hline \multicolumn{7}{|l|}{$F A D 3$-silenced ${ }^{\mathrm{b}}$} \\
\hline Root & $30.4 \pm 0.3$ & NA & $6.2 \pm 0.7$ & $3.0 \pm 0.4$ & $42.8 \pm 1.2$ & $17.4 \pm 1.3$ \\
\hline Stem & $24.8 \pm 0.3$ & $0.43 \pm 0.3$ & $6.5 \pm 0.3$ & $4.4 \pm 0.1$ & $33.4 \pm 2.5$ & $30.3 \pm 1.6$ \\
\hline Leaf & $18.8 \pm 0.3$ & $2.9 \pm 0.2$ & $5.3 \pm 0.2$ & $2.5 \pm 0.1$ & $25.0 \pm 1.4$ & $45.2 \pm 1.7$ \\
\hline Petiole & $21.3 \pm 0.4$ & $0.03 \pm 0.0$ & $7.6 \pm 0.1$ & $2.6 \pm 0.2$ & $37.8 \pm 0.5$ & $30.5 \pm 1.2$ \\
\hline Immature seed & $11.4 \pm 0.8$ & $0.2 \pm 0.1$ & $4.6 \pm 0.4$ & $8.3 \pm 0.4$ & $36.5 \pm 1.1$ & $25.1 \pm 2.6$ \\
\hline Mature seed & $15.3 \pm 0.4$ & NA & $2.6 \pm 0.05$ & $16.1 \pm 0.3$ & $56.9 \pm 0.4$ & $9.1 \pm 0.2$ \\
\hline
\end{tabular}

\footnotetext{
a Amount \pm standard deviation $(n=3)$; NA = not detected at significant levels.

b Plants silenced for omega-3 fatty acid desaturase (FAD3) gene GmFAD3.
} 
twice as much JA as mock-inoculated plants, indicating that BPMV infection increased JA accumulation in soybean (Fig. 4A). Furthermore, JA levels in GmFAD3-silenced plants were even higher than in vector-infected plants; GmFAD3-silenced plants contained approximately two- or fivefold more JA compared with vector-infected or mock-treated plants, respectively (Fig. 4A).

JA is known to induce the differential expression of defense related genes, such as the pathogenesis-related $(P R)$ genes, including PRP and PR3 in soybean (Browse 2009; Creelman et al. 1992; Mazarei et al. 2007; Penninckx et al. 1998). Therefore, we analyzed the expression of these genes in the mockor vector-treated and GmFAD3-silenced plants using Northern blot analysis. Consistent with our previous report (Kachroo et al. 2008), BPMV infection did not significantly alter $P R$ gene expression (Fig. 4B). In contrast, the GmFAD3-silenced plants exhibited increased expression of $P R 3$ and $P R P$.

JA is well known to antagonize signaling mediated by the defense-related phytohormone salicylic acid (SA), and some plant pathogens mimic this response to suppress host defense (Brooks
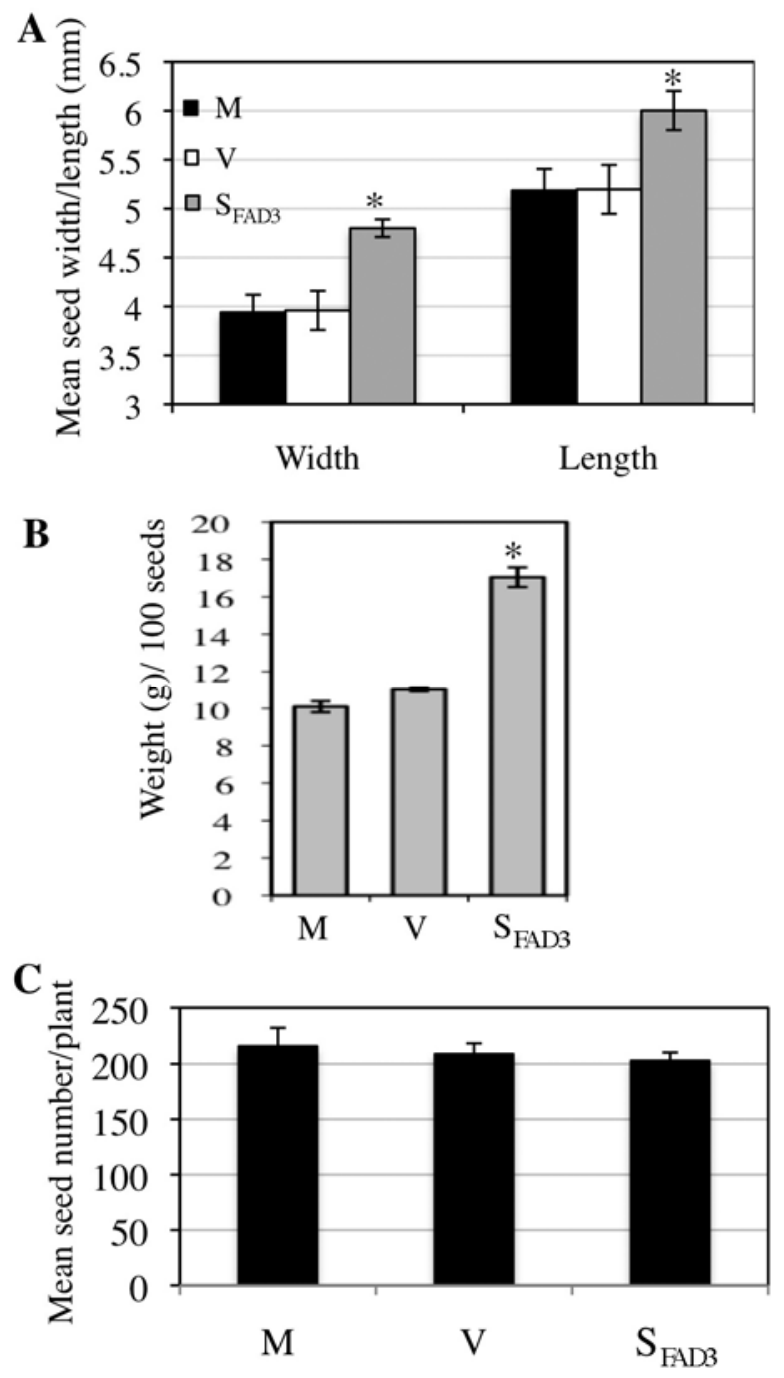

Fig. 2. Silencing Glycine max omega-3 fatty acid desaturase (GmFAD3) genes enhances seed yield in soybean. A, Histogram of mean seed width and length. B, Histogram of weight in grams per 100 seeds. C, Histogram of mean number of seed from mock-inoculated (M) or vector-infected (V) and $G m F A D 3$-silenced $\left(\mathrm{S}_{\mathrm{FAD} 3}\right)$ plants. Asterisks denote data statistically significant from $\mathrm{M}$ and $\mathrm{V}$, as determined by Student's $t$ test. A, $P<0.001$, $n=100 ; \mathbf{B}, P<0.0001, n=5$. Data are representative of four independent experiments with eight to 10 plants tested per experiment. et al. 2005; Cui et al. 2005; Zhao et al. 2003). For example, the bacterial pathogen Pseudomonas syringae produces the phytotoxin coronatine, a structural analogue of JA. The pathogen gains virulence when coronatine mimics JA to inhibit SA-derived defenses in the host (Brooks et al. 2005). Therefore, we tested whether increased JA accumulation in the GmFAD3silenced plants altered their response to $P$. syringae. Vector-infected and GmFAD3-silenced plants (cv. Essex) were inoculated with a virulent strain of $P$. syringae pv. glycinea. Bacterial proliferation was monitored at 0 and 4 days postinoculation (dpi). As expected, the GmFAD3-silenced plants showed slightly enhanced susceptibility to virulent $P$. syringae $(P<0.001, n=4)$, consistently accumulating approximately threefold higher bacteria than vector-infected plants (Fig. 4C). Together, these results showed that the GmFAD3-silenced plants accumulated higher levels of JA, expressed increased levels of JA-responsive $P R$ genes, and accumulated slightly more virulent $P$. syringae.

\section{Exogenous application of JA increases accumulation of BPMV in soybean.}

The fact that infection of soybean plants with the BPMV vector alone induced JA accumulation suggested that the higher levels of JA in the $G m F A D 3$-silenced plants were likely the result of the higher BPMV titer in these plants. However, it was also possible that the increased JA in the GmFAD3silenced plants led to the increase in virus titer. To test the possibility that increased JA in the GmFAD3-silenced plants contributed to the enhanced accumulation of BPMV, we treated healthy soybean plants (cv. Essex) with exogenous JA, followed by inoculation with BPMV (empty BPMV RNA2-based
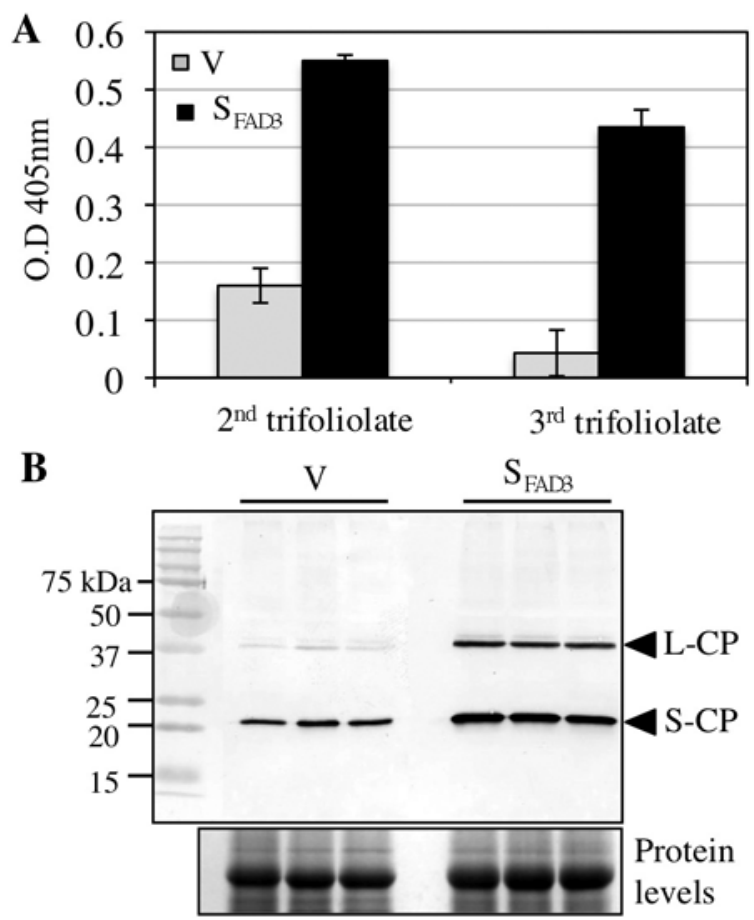

Fig. 3. Glycine max omega-3 fatty acid desaturase (GmFAD3)-silenced $\left(\mathrm{S}_{\mathrm{FAD} 3}\right)$ plants accumulate increased levels of Bean pod mottle virus (BPMV). A, Enzyme-linked immunosorbent assay showing BPMV levels in the second and third trifoliolates of vector-infected (V, gray bars) or $\mathrm{S}_{\mathrm{FAD} 3}$ (black bars) plants at 14 days postinoculation (dpi). Error bars indicate standard deviation, $n=4$. $\mathbf{B}$, Western blot analysis showing levels of large (L, 42-kDa) and small (S, 21-kDa) coat protein (CP) of BPMV (upper panel) in $10 \mu \mathrm{g}$ of total leaf protein extracts from $\mathrm{V}$ and $\mathrm{S}_{\mathrm{FAD} 3}$ plants at 10 dpi. BPMV CPs detected using CP-specific antibodies. Lower panel: protein-loading control; Coomassie blue staining of the approximately 49$\mathrm{kDa}$ band on the corresponding gel. 
vector plus RNA1). Northern blot analysis of $P R 3$ expression was used to test the efficacy of JA treatment (data not shown). BPMV accumulation in the JA-pretreated plants was analyzed using ELISA and Western blot analysis and compared with that in water-treated plants. ELISA of BPMV levels at 1, 2, and 3 dpi showed that the JA-pretreated plants accumulated significantly higher levels of BPMV than the water-pretreated control in their inoculated tissues at 2 dpi (Fig. 5A). Furthermore, BPMV was detected much earlier (within $3 \mathrm{dpi}$ ) and accumulated at much higher levels in the systemic tissues of plants pretreated with JA (Fig. 5B). In contrast, BPMV was only readily detected at $7 \mathrm{dpi}$ in the systemic tissues of waterpretreated plants. These results were confirmed by Western blot analysis, which detected L-CP and S-CP of BPMV in the systemic tissues of JA-pretreated plants but not in the waterpretreated plants at $3 \mathrm{dpi}$. The JA-pretreated plants also contained higher levels of BPMV at 7 dpi (Supplementary Fig. 4). We next tested whether exogenous JA altered BPMV accumulation when applied post-BPMV inoculation. Soybean plants (cv. Essex) were first inoculated with BPMV and then treated with exogenous water or JA at 12 or $24 \mathrm{~h}$ post-BPMV inoculation. ELISA analysis did not detect significant differences in

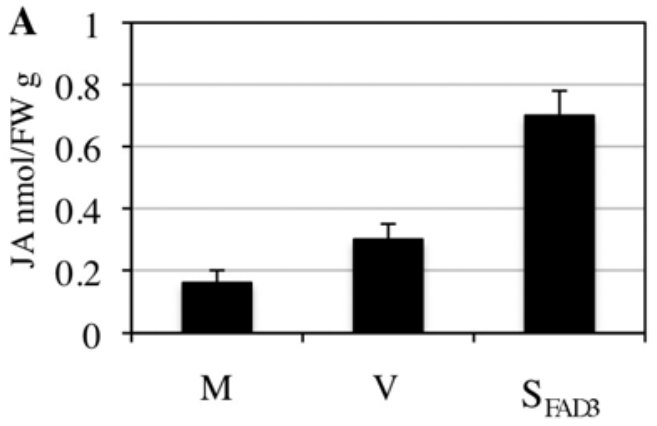

B
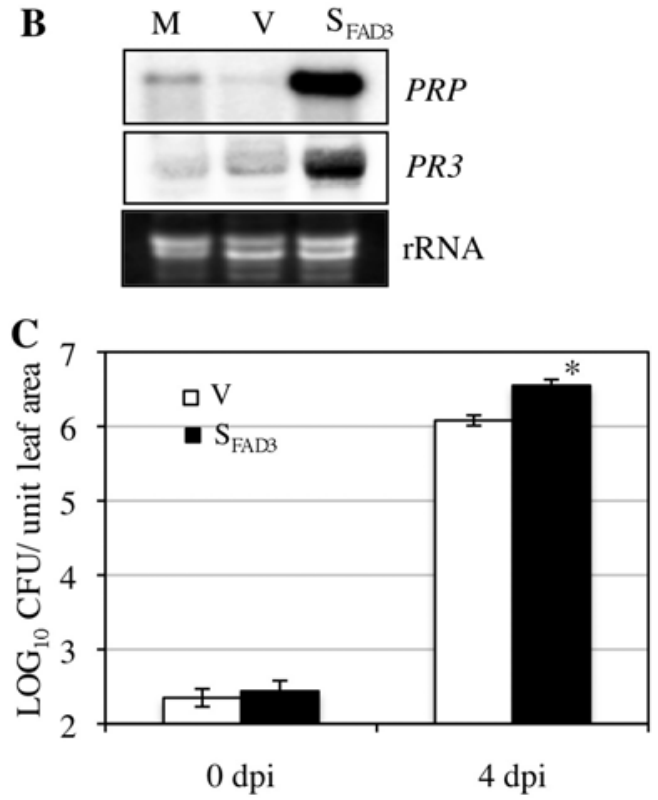

Fig. 4. Glycine max omega-3 fatty acid desaturase (GmFAD3) $\left(\mathrm{S}_{\mathrm{FAD} 3}\right)$ silenced plants contain high endogenous levels of jasmonic acid (JA). A, JA levels per gram fresh weight $(\mathrm{FW})$ of mock-inoculated (M), vectorinfected $(\mathrm{V})$, and $\mathrm{S}_{\mathrm{FAD} 3}$ plants. B, Northern blot analysis showing expression of $P R P$ and $P R 3$ in $\mathrm{M}, \mathrm{V}$, and $\mathrm{S}_{\mathrm{FAD} 3}$ plants. Ethidium bromide staining of rRNA was used as a loading control. $\mathbf{C}, \log _{10}$ value of a virulent strain of Pseudomonas syringae counts in $\mathrm{V}$ (white bars) and $\mathrm{S}_{\mathrm{FAD} 3}$ (black bars) plants. CFU per unit leaf area from infected leaves at 0 and 4 days postinoculation (dpi) are presented. Error bars indicate standard deviation, $n=5$. Student's $t$ test was used to determine statistical significance. Asterisk denotes significant difference from $\mathrm{V}$ plants $(P<0.001)$.
BPMV levels in the inoculated tissues of plants post-treated with water or JA (Fig. 5C, data shown for 3 dpi). In contrast, BPMV accumulated to approximately sevenfold higher levels in the systemic tissues of plants post-treated with JA compared with those post-treated with water (Fig. 5D, data shown for 7 dpi). Together, these results showed that exogenous JA enhanced BPMV accumulation in soybean.

\section{JA-derived increase in BPMV levels is not related to the inhibition of $\mathrm{SA}$ accumulation or $\mathrm{SA}$ responsiveness in the GmFAD3-silenced plants.}

JA is well known to antagonize SA-mediated defense responses in plants (Kachroo and Kachroo 2007). Therefore, we tested whether the JA-derived increased accumulation of BPMV in the GmFAD3-silenced plants was associated with a defect in SA accumulation or responsiveness in these plants. Analysis of SA levels showed that BPMV infection increased SA levels in soybean, which is consistent with our previous report (Kachroo et al. 2008). Interestingly, the total SA in the GmFAD3-silenced plants was even higher (approximately 1.6fold) than those in vector-infected plants (Fig. 6A). We next determined whether the GmFAD3-silenced plants exhibited enhanced expression of the SA-inducible PRI gene (Mazarei et al. 2007) in response to their enhanced endogenous SA levels. Northern blot analysis showed that $P R I$ was, indeed, induced in the GmFAD3-silenced plants (Fig. 6B). This indicated that the plants were not defective in SA accumulation or SA-induced defense gene expression.

Previously, we showed that soybean plants containing high levels of SA exhibit enhanced resistance to the oomycete pathogen Phytophthora sojae (Kachroo et al. 2008). To test whether the increased SA in the GmFAD3-silenced plants also enhanced resistance to $P$. sojae, vector-infected and GmFAD3-silenced plants (cv. Essex) were inoculated with race 3 of $P$. sojae, which is virulent on the Essex cultivar. As expected, vectorinfected plants produced necrotic lesions at the inoculation site, which increased in size progressively. The vector-infected plants rapidly succumbed to $P$. sojae infection, such that $100 \%$ of inoculated plants were dead by $7 \mathrm{dpi}$ (Fig. 6C and D). In comparison, the GmFAD3-silenced plants produced much smaller lesions, which were greatly restricted in their spread (Supplementary Fig. 5). Furthermore, the GmFAD3-silenced plants consistently showed greatly enhanced resistance to $P$. sojae; nearly $90 \%$ of the inoculated GmFAD3-silenced plants survived infection by $7 \mathrm{dpi}$. These results showed that the high SA-accumulating GmFAD3-silenced plants showed enhanced basal resistance to $P$. sojae.

\section{SA levels in soybean do not affect BPMV accumulation.}

To test whether SA levels affected soybean basal resistance to BPMV, we treated healthy soybean plants with water or SA. As expected, SA treatment induced PRI expression (data not shown). SA- and water-treated plants were then inoculated with BPMV (empty BPMV RNA2-based vector plus RNA1) and virus levels monitored in the inoculated and systemic tissues of these plants. Western blot analysis did not detect significant differences in the levels of BPMV in leaf extracts from inoculated tissues at $3 \mathrm{dpi}$ or systemic tissues at $7 \mathrm{dpi}$ between the water- or SA-treated plants (Fig. 7A). ELISA analysis confirmed that the SA-treated plants accumulated levels of BPMV comparable with the water-treated control plants in inoculated as well as systemic tissues (Fig. 7B and C). These results indicated that increased levels of SA did not alter soybean response to BPMV.

Next, we generated soybean plants that were defective in SA synthesis and assessed their response to BPMV. The SA-synthesizing phenylalanine ammonia lyase $(P A L)$ gene $(G m P A L$, X52953) of soybean was silenced using the BPMV-based vector. 
A recombinant vector containing a 363-bp fragment (nucleotide positions 28 to 390) of GmPAL was generated. Soybean plants (cv. Essex) were inoculated with the vector-inoculated control or recombinant vector targeting GmPAL. RT-PCR analysis showed that $G m P A L$ was effectively silenced in the $G m P A L$-silenced plants (Fig. 8A). Analysis of SA levels showed that the GmPALsilenced plants contained significantly reduced SA and SA-glucoside (SAG) compared with vector-infected plants (Fig. 8B). Inoculation with virulent Pseudomonas syringae resulted in approximately threefold increase in SA or SAG levels in mocktreated and vector-infected plants. However, GmPAL-silenced plants were unable to accumulate SA or SAG in response to pathogen infection. Correspondingly, these plants also accumulated slightly more virulent $P$. syringae than control plants (Fig. 8C). The GmPAL-silenced plants consistently accumulated threefold more bacteria than the vector-infected plants $(P<$ $0.001, n=5)$. These results showed that silencing GmPAL inhibited the plant's ability to induce SA-responsive defense signaling. To test whether the inability to accumulate SA altered defense against BPMV, we compared BPMV levels in GmPAL-silenced versus vector-infected plants. ELISA analysis showed that the GmPAL-silenced plants accumulated levels of BPMV similar to the vector-infected plants (Fig. 8D). This was confirmed by Western blot analysis, which showed similar levels of the BPMV L-CP and S-CP in leaf extracts from both vector-infected and GmPAL-silenced plants (data not shown). Together, these results showed that the inability to accumulate SA via the PAL pathway did not alter BPMV accumulation in soybean.

\section{DISCUSSION}

The production of soybean cultivars with oil containing low levels of 18:3 is a major goal of the soybean industry. In addition to improving oil stability and flavor, lowering 18:3 is de-
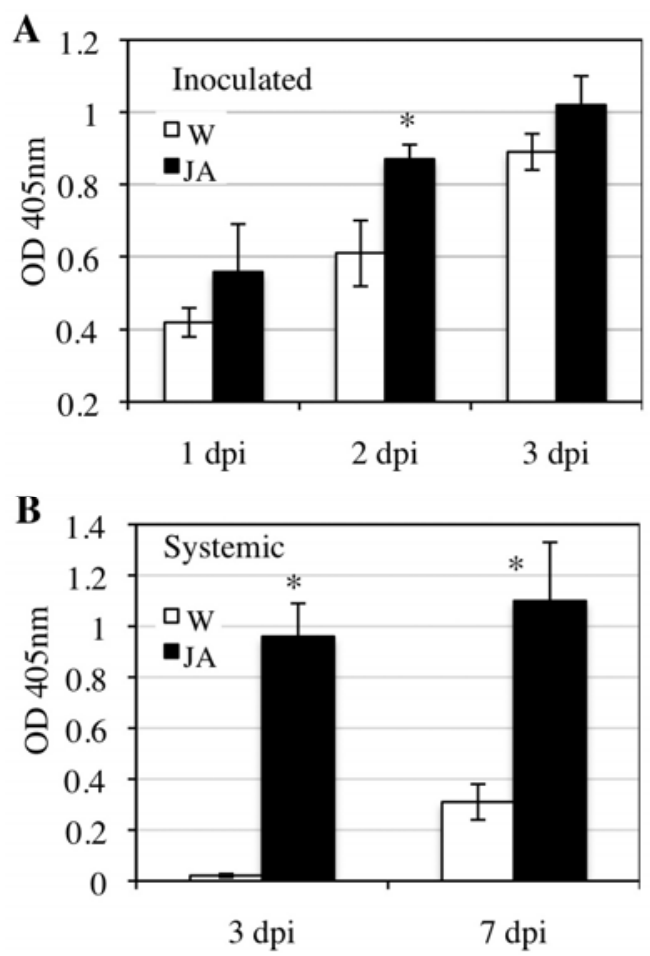

sirable from the health point of view. In soybean, three microsomal isoforms of GmFAD3 contribute to the conversion of 18:2 to 18:3. In this study, we used a BPMV-based vector to efficiently silence genes encoding the three isoforms in nonseed tissues. Surprisingly, we discovered that the GmFAD3silenced plants produced seed of larger size and weight than control plants and accumulated higher levels of BPMV and the phytohormones SA and JA. The observed phenotypes are of considerable interest because they suggest a role for SA or JA in regulating seed development and a specific role for JA in soybean defense to BPMV.

BPMV infection of field-grown soybean reduces seed yield and quality and delays plant maturation (Myhre et al. 1973; Ross 1968). Seed from greenhouse-grown vector-infected and GmFAD3-silenced plants also showed seed coat mottling symptoms and produced fewer three-seeded pods (this study). Although a reduction in three-seeded pods is undesirable, the total seed number from $G m F A D 3$-silenced plants was not significantly lower than that from vector-infected plants, because the GmFAD3-silenced plants produced a higher number of one-seeded pods. Furthermore, GmFAD3-silenced plants also produced significantly larger seed, resulting in an approximately $55 \%$ gain of seed weight in greenhouse-grown plants. Thus, in addition to enhancing BPMV accumulation, silencing GmFAD3 also enhanced soybean yield by increasing seed size. This is surprising, given the fact that BPMV infection generally lowers field-grown soybean yield in the range of 3 to $52 \%$ (Ross 1968; Schwenk and Nickell 1980). Clearly, though, it is not the increased accumulation of BPMV that enhances seed size, because no significant changes were observed in the size of seed obtained from greenhouse-grown vector-infected plants. Increased seed size has not been reported in the Arabidopsis fad3 mutant, which carries a genetic mutation in the FAD3 gene (Browse et al. 1993). This may be because Arabidopsis
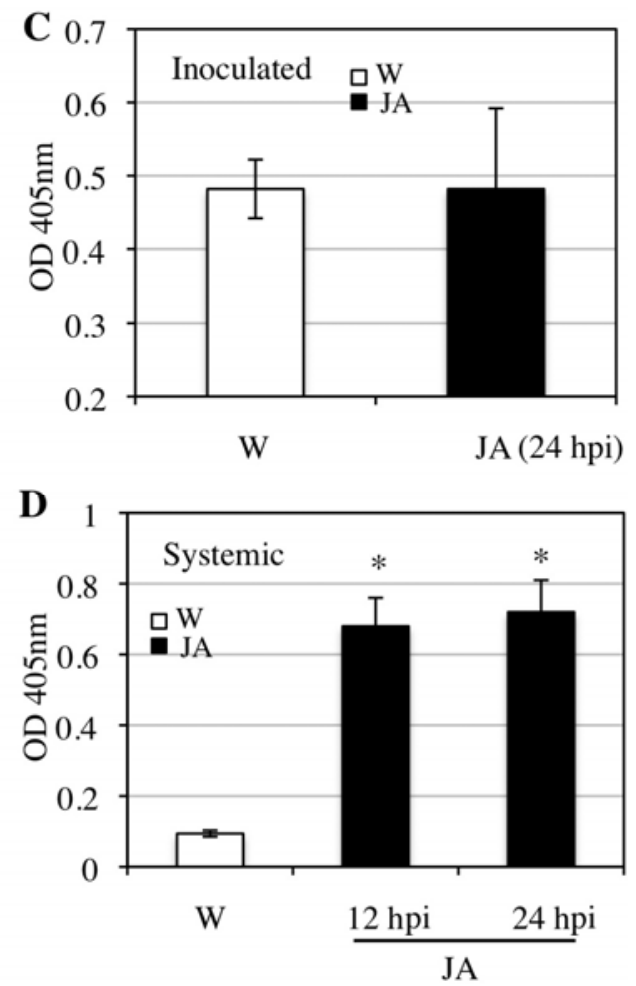

Fig. 5. Exogenous application of jasmonic acid (JA) enhances Bean pod mottle virus (BPMV) accumulation in soybean. Enzyme-linked immunosorbent assay of BPMV levels in the A and C, inoculated or B and D, systemic leaves of water (W, white bars)- or JA (black bars)-treated plants at various days postinoculation (dpi) with BPMV. A and B, Plants were treated with JA first, followed by inoculation with BPMV 48 h later. C and D, Plants were inoculated with BPMV first, then treated with JA at 12 or $24 \mathrm{~h}$ postinoculation (hpi) with BPMV. BPMV levels in C, inoculated leaves at 3 dpi and D, systemic leaves at $7 \mathrm{dpi}$ are shown. Error bars indicate standard deviation, $n=4$. Asterisks denote data significantly different from $\mathrm{W}(P<0.005)$. 
and soybean differ significantly in their seed development processes. However, RNAi-mediated silencing of GmFAD3 in a seed-specific manner was also not reported to alter yield in soybean, even though the data presented in that report do show a slight increase in yield (Flores et al. 2008). One possibility is that silencing GmFAD3 in the growing plant alters different physiological processes than those in seed-specific RNAi lines (Flores et al. 2008). Testing this possibility would require genome-wide transcriptional profiling followed by targeted gene function analyses. A second possibility is that GmFAD3 plants are altered in nontarget gene expression. Preliminary analyses showed that the expression of three other closely related sequences (putative FAD3s Glyma03g07570, Glyma07g18350, and Glymallg27190) was indeed reduced, but only very minimally, in the GmFAD3-silenced plants (Supplementary Fig. 6). The expression (albeit reduced) of these putative FAD3s might explain why only moderate changes in 18:2 and 18:3 levels were detected in the GmFAD3-silenced plants.

The high SA in the GmFAD3-silenced plants is unlikely to be the cause of their increased seed size, because this phytohormone usually activates energy-intensive, defense-related processes in the plant (Vlot et al. 2009). Indeed, SA-responsive $P R$ genes and basal resistance to Phytophthora sojae are heightened in the GmFAD3-silenced plants. Interestingly, though, the GmFAD3-silenced plants accumulate slightly more virulent Pseudomonas syringae than the control plants, suggesting that the increased JA might suppress SA-inducible basal defenses. Indeed, the JA-mediated antagonism of SA-dependent defense responses is well known to suppress basal defense against $P$. syringae (Brooks et al. 2005; Kloek et al. 2001; Zhao et al. 2003). However, it appears that certain threshold levels of JA may be required for this inhibition, because vector-infected plants, whose JA levels are higher than mocktreated plants but lower than the GmFAD3-silenced plants, do not accumulate increased $P$. syringae. Whether the increased JA levels in the GmFAD3-silenced plants contribute to the increased seed size is not known. Although JA is essential for pollen development and fertility in Arabidopsis, it has not been associated with increased seed size (Browse 2005). However, exogenous application of JA, in the absence of drought stress, was reported to enhance grain yield in amaranth (Delano-Frier et al. 2004). On the other hand, increased production of JA in transgenic rice plants during drought stress resulted in a loss in grain yield (Kim et al. 2009). Further characterization of the various metabolic changes in the GmFAD3-silenced plants could help elucidate the role of JA, if any, in yield increase.

Whereas increased accumulation of SA in response to BPMV is consistent with many other compatible plant-virus interactions, the increased accumulation of JA in BPMV-infected soybean plants is an unexpected finding (Lewsey et al. 2010).

\section{A}

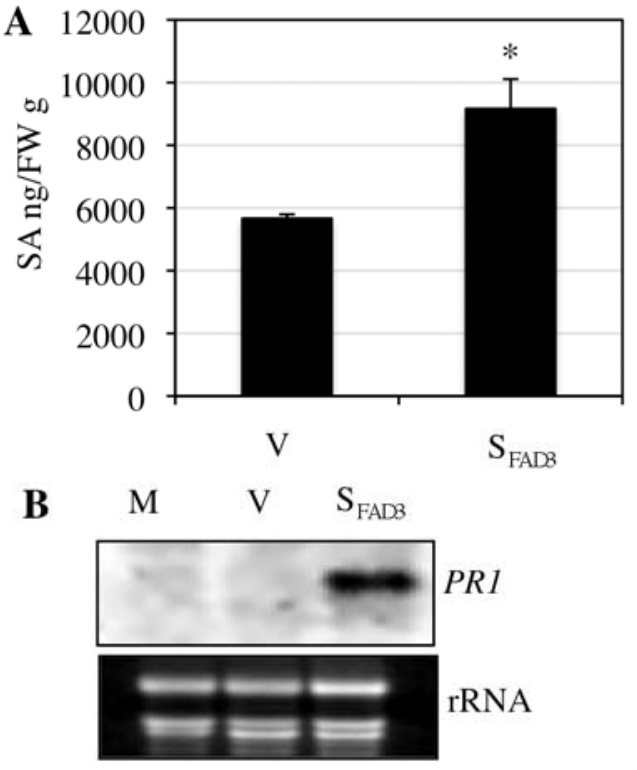

C

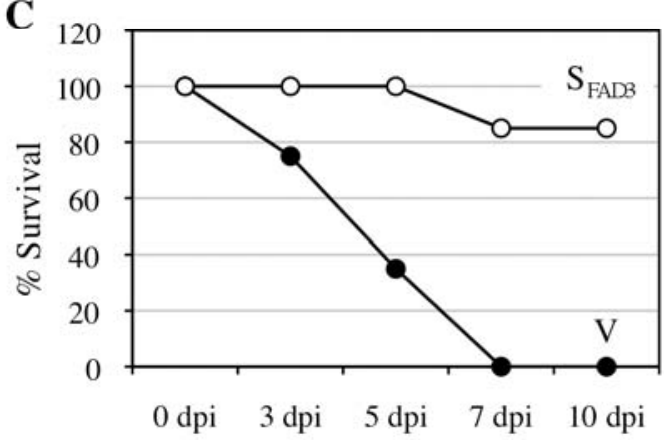

D
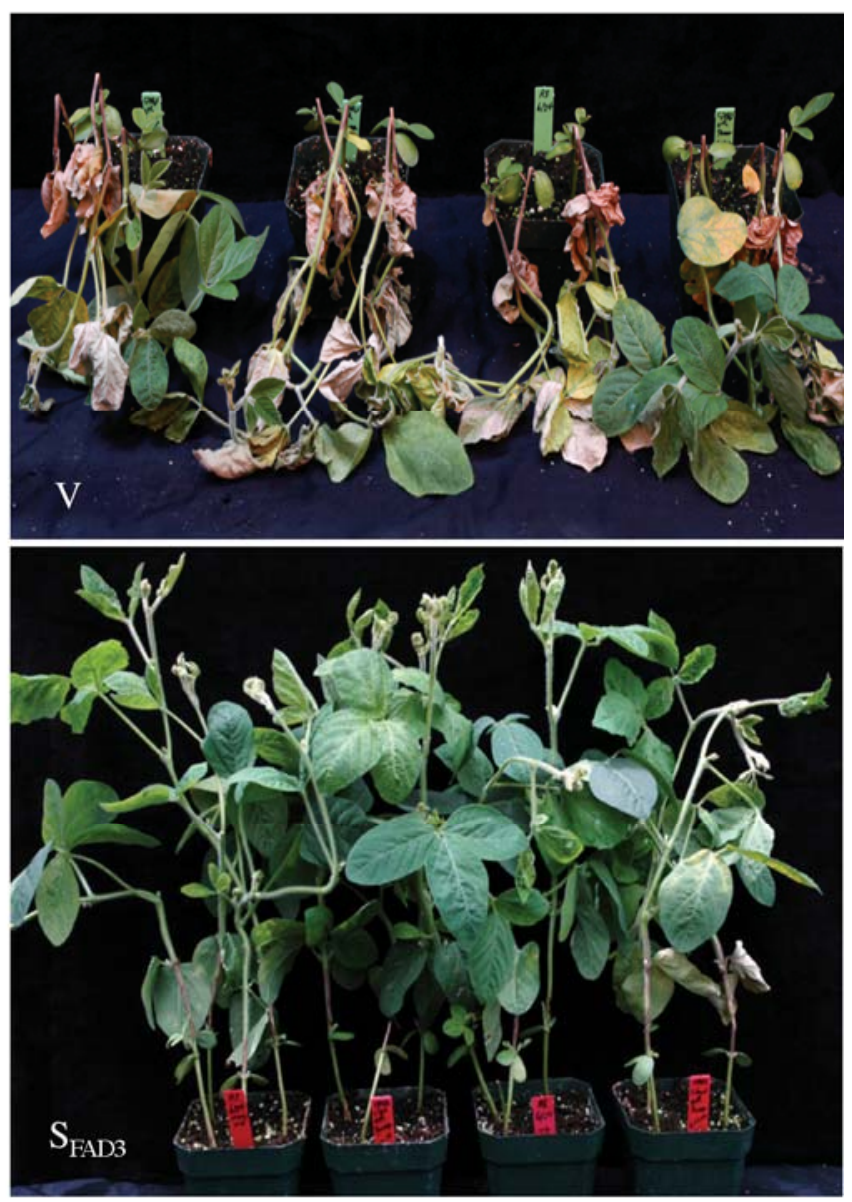

Fig. 6. Salicylic acid (SA) levels in Glycine max omega-3 fatty acid desaturase (GmFAD3)-silenced ( $\left.\mathrm{S}_{\mathrm{FAD} 3}\right)$ plants and the effect of exogenous SA on soybean defense responses. A, Total SA levels of vector-inoculated (V) or $\mathrm{S}_{\mathrm{FAD} 3}$ plants. SA levels were measured from leaves of the second trifoliolate, at 17 days postinoculation (dpi) with the respective Bean pod mottle virus-based vector and presented as per gram fresh weight (FW). Asterisk denotes data significantly different from V $(P<0.005)$. B, Northern blot analysis showing expression of $P R 1$ in mock-inoculated (M), V, and $\mathrm{S}_{\mathrm{FAD}}$ plants. Ethidium bromide staining of rRNA was used as a loading control. C, Percent survival of V (closed circles) and $\mathrm{S}_{\mathrm{FAD} 3}$ (open circles) plants (cv. Essex), at 0, 3, 5, 7, and 10 dpi with Phytophthora sojae (race 3). Results are representative of three independent experiments with 15 to 20 plants tested per experiment. D, Morphology of $\mathrm{V}$ and $\mathrm{S}_{\mathrm{FAD} 3}$ plants at 14 dpi with $P$. sojae. 
Clearly, BPMV infection increases JA levels in soybean; vectorinfected plants contain higher JA than mock-treated plants. However, increased JA could also be facilitating BPMV accumulation in the GmFAD3-silenced plants. Indeed, JA application, either before or after BPMV inoculation, enhanced virus accumulation. This suggests that silencing GmFAD3 enhances BPMV accumulation because it increases endogenous JA levels. However, unlike in the case of $P$. syringae, this is likely not associated with a suppression of SA-derived defenses, because exogenous application of SA does not alter soybean response to BPMV. Moreover, silencing the GmPAL gene, which lowers pathogen-responsive SA accumulation and promotes the accumulation of $P$. syringae, does not affect BPMV accumulation in soybean. Nevertheless, the possibility that JA antagonizes the isochorishmate synthase-derived SA pathway to affect BPMV levels cannot be ruled out in this study.

JA is well known to mediate plant defense against herbivores and necrotrophic pathogens but a role for JA in defense to viruses is not known (Browse 2009). Indeed, viral resistance is generally not associated with JA (Kachroo et al. 2000; Ton et al. 2002). Although some viruses do induce JA-responsive gene expression others, such as Cucumber mosaic virus, actually inhibit JA-dependent signaling without altering JA biosynthesis in the plant (Lewsey et al. 2010; Love et al. 2005; Yang et al. 2008). It has been suggested that this response may be linked to the importance of JA in plant defense to arthropod herbivores, many of which serve as vectors for transmitting plant viruses, including BPMV (Browse and Howe 2008; Fulton et al. 1980; Howe and Jander 2008). It is quite likely that, whereas some viruses evolved to promote their own spread by suppressing host defense against the insect vector, others such as BPMV evolved to utilize the plant's defense response to the vector for promoting its own accumulation. Whether the cause or the result of increased phytohormone accumulation, the association of JA with enhanced susceptibility to BPMV provides an exciting avenue for examining the mechanisms underlying soybean defense to this virus and the role of JA in plant defense to viruses. This is particularly important considering that all known soybean cultivars lack resistance to BPMV.

\section{MATERIALS AND METHODS}

\section{Plant growth conditions, seed yield, and chemical treatments.}

Soybean (G. $\max$ (L.) Merr.) cvs. Harosoy and Essex were grown in the greenhouse with day and night temperatures of 25 and $20^{\circ} \mathrm{C}$, respectively. For silencing experiments, inoculation of recombinant BPMV vectors and confirmation of silencing was carried out as described before (Kachroo et al. 2008). For seed-yield-related traits, results presented are representative of seed measurements from four independent silencing experiments. At least 8 to 10 individual plants were tested per genotype per experiment. Results presented in Figure 2 are from plants of cv. Essex. Increase in seed weight was also confirmed in two independent experiments in cvs. Harosoy, Harosoy 63, Clark, and Merit.

\section{Construction of viral vectors,}

in vitro transcription, and plant inoculation.

Generation of BPMV-based silencing vector, in vitro transcription, and rub-inoculation of soybean leaves was carried out as before (Kachroo et al. 2008; Zhang and Ghabrial 2006). A 372-bp fragment (nucleotide positions 424 to 795) from $G m F A D 3 A$ was used to generate a vector targeting $G m F A D 3 A$, $G m F A D 3 B$, and $G m F A D 3 C$. A 363-bp fragment (nucleotide positions 28 to 390 ) from $G m P A L$ was used to silence this gene.
RNA extraction and Northern and RT-PCR analysis.

RNA from leaf tissues of soybean plants at the V1/V2 growth stage was extracted using the TRIzol reagent (Invitrogen, Carlsbad, CA, U.S.A.) per the manufacturer's instructions. Northern blot analysis and synthesis of random-primed probes were performed as described before (Kachroo et al. 2001). RT and first-strand cDNA synthesis was carried out using Superscript II (Invitrogen). Two to three independent RNA preparations were analyzed at least twice by RT-PCR using a reduced number of cycles (15 to 20) for evaluating relative differences in transcript levels. Primers were designed for full-length coding sequences available in the database, including GmFAD3A (AY204710), GmFAD3B (AY204711), GmFAD3C (AY204712), PR1 (AI930866), PRP (AF248055), PR3 (AF202731), and $\beta$ -

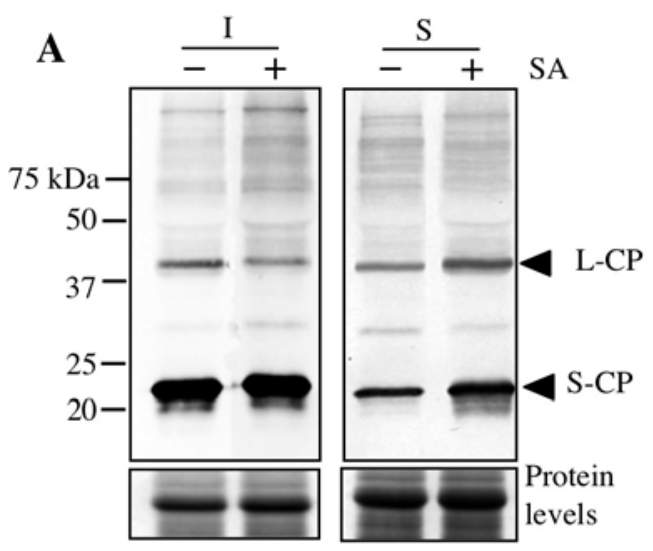

B
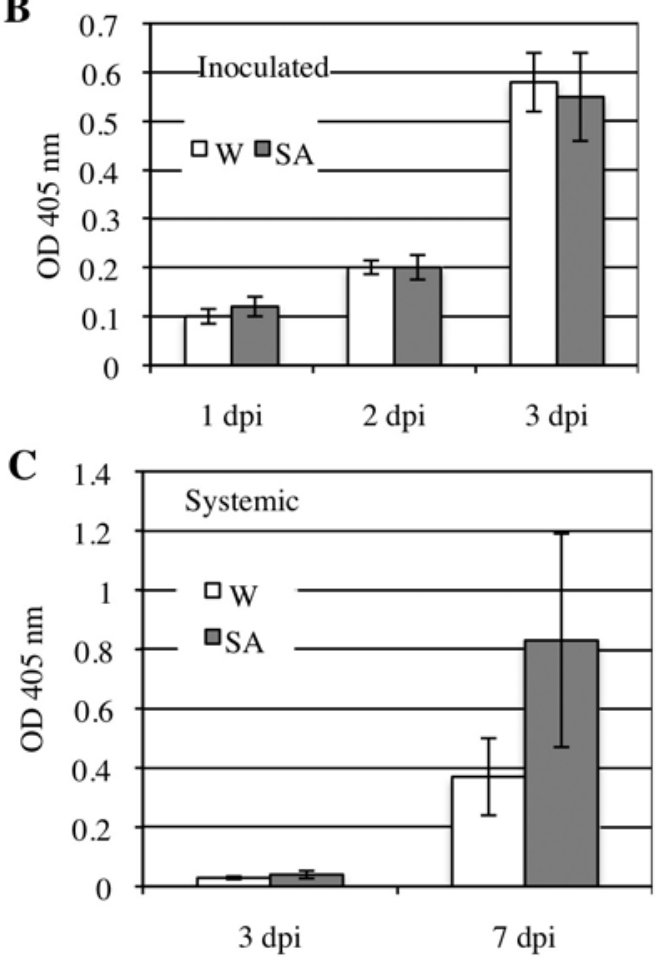

Fig. 7. Exogenous salicylic acid (SA) application does not alter Bean pod mottle virus (BPMV) accumulation in soybean. A, Western blot analysis showing BPMV large (L, 42-kDa) and small $(\mathrm{S}, 21-\mathrm{kDa})$ coat proteins (CP) in the inoculated leaves (I) at 3 days postinoculation (dpi) or systemic leaves (S) at 7 dpi of plants treated with water $(\mathrm{W},-)$ or SA (+). Lower panel: protein-loading control; Coomassie blue staining of the approximately $49-\mathrm{kDa}$ band on the corresponding gel. Enzyme-linked immunosorbent assay of BPMV levels in the $\mathbf{B}$, inoculated or $\mathbf{C}$, systemic leaves of W (white bars)- or SA (gray bars)-treated plants at 1,2,3, or 7 dpi. Error bars indicate standard deviation, $n=4$. 
tubulin (M21297). Glyma01g29630, Glyma03g07570, Glyma$07 \mathrm{~g} 18350$, and Glyma11g27190 sequences were obtained from the soybean genome database.

Plant protein extraction, immunoblot analysis, and ELISA.

Proteins were extracted in $10 \mathrm{mM} \mathrm{NaHPO}$ buffer, pH 6.8, as described previously (Reddy et al. 2001). Protein concentration was measured by the Bio-RAD protein assay (Bio-Rad, Hercules, CA, U.S.A.). Proteins (30 to $50 \mu \mathrm{g}$ ) were fractionated on 7 to $10 \%$ sodium dodecyl sulfate polyacrylamide gel electrophoresis gels and subjected to immunoblot analysis using anti-BPMV-CP antibodies (Ghabrial and Schultz 1983) and the protocol of Huang and associates (1997). ELISA was performed as described by Ghabrial and Schultz (1983).

\section{Pathogen inoculations.}

$P$. syringae inoculation of soybean and monitoring of bacterial proliferation was carried out as described before ( $\mathrm{Fu}$ et al. 2009). Mock inoculations were carried out with $10 \mathrm{mM} \mathrm{MgCl}_{2}$ in $0.04 \%$ Silwett L-77. Experiments were repeated three to four times. For Phytophthora sojae (race 3), inoculations was grown on V8 agar at $25^{\circ} \mathrm{C}$ in the dark. $P$. sojae infections were carried out as described before (Kachroo et al. 2008). Experiments were repeated three times with 15 to 20 plants tested per silenced line per experiment.

\section{SA, JA, and FA analyses.}

$\mathrm{SA}$ and SAG were extracted and measured from $0.3 \mathrm{~g}$ of fresh weight leaf tissue, as described before (Chandra-Shekara et al. 2006). FA analysis was carried out as described previously (Kachroo et al. 2008). For FA profiling, one or a few leaves of 4-week-old plants were placed in $2 \mathrm{ml}$ of $3 \% \mathrm{H}_{2} \mathrm{SO}_{4}$ in methanol containing $0.001 \%$ butylated hydroxytoluene. After $30 \mathrm{~min}$ of incubation at $80^{\circ} \mathrm{C}, 1 \mathrm{ml}$ of hexane with $0.001 \%$ butylated hydroxytoluene was added. The hexane phase was then transferred to vials for gas chromatography (GC), and 1$\mu \mathrm{l}$ samples were analyzed by GC on a Varian FAME $0.25-\mathrm{mm}$ $650-\mathrm{m}$ column and were quantified with flame ionization detection. The identities of the peaks were determined by comparing the retention times with known FA standards. Mole values were calculated by dividing peak area by molecular weight of the FA. For JA extraction, $1 \mathrm{~g}$ of leaf tissue was ground in liquid nitrogen and extracted in 100\% methanol using dihydroJA as an internal standard.

\section{SA and JA treatments.}

For JA treatment, plants at the VC stage were sprayed with $50 \mu \mathrm{M}$ solution prepared in sterile water and covered with a transparent plastic dome for $48 \mathrm{~h}$ to maximize exposure to JA. For SA treatment, plants at the VC stage were sprayed with a 1-mM benzothiadiazole $S$-methyl ester solution prepared in sterile water for two consecutive days.

\section{ACKNOWLEDGMENTS}

We thank W. Havens for technical assistance, J. Johnson for help with FA analysis, A. Crume for maintaining plants, and P. Kachroo for critical review of the manuscript. This work was supported by grants from the
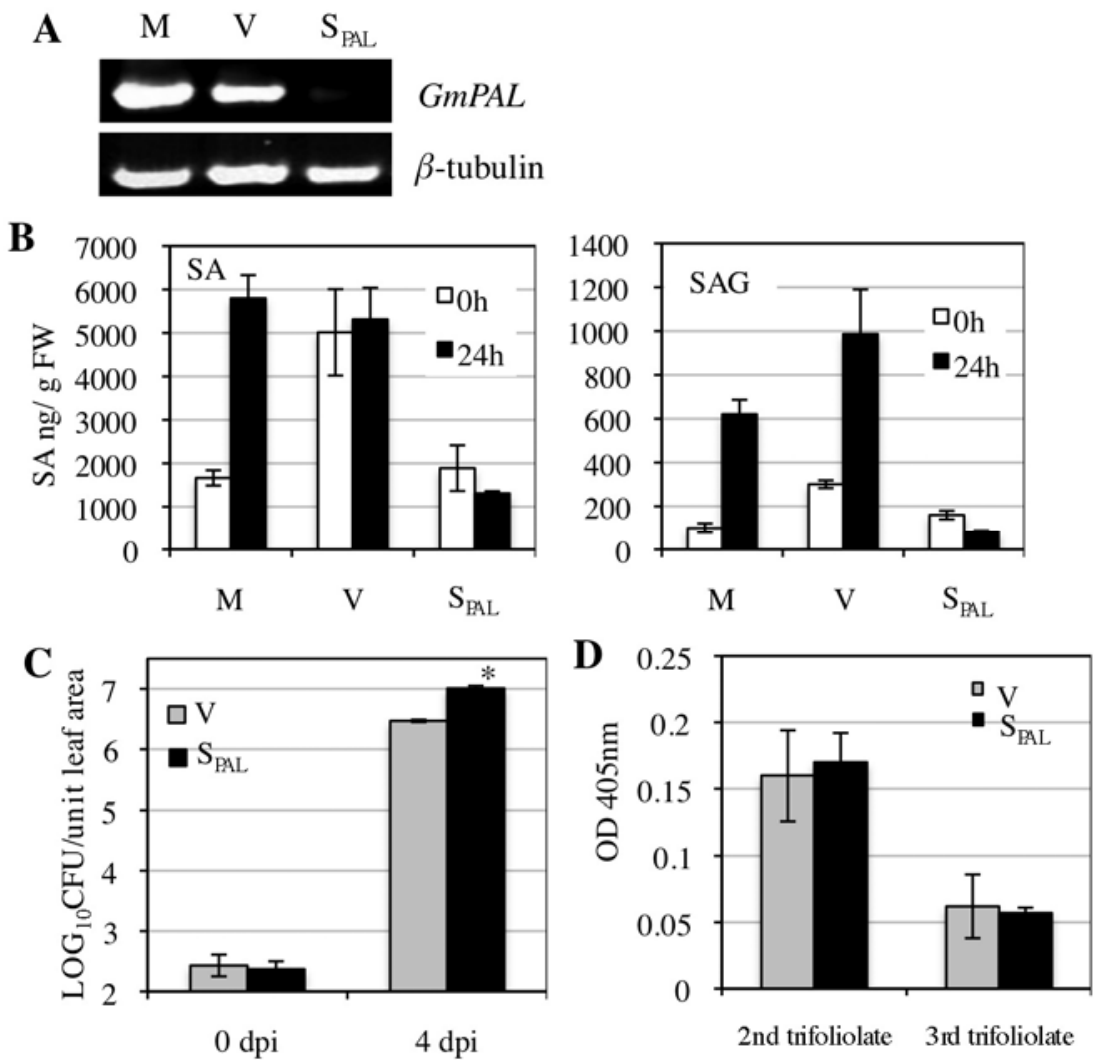

Fig. 8. Silencing the phenylalanine ammonia lyase (GmPAL) gene does not alter Bean pod mottle virus (BPMV) accumulation in soybean. A, Reverse-transcription polymerase chain reaction analysis showing expression of $G m P A L$ in mock-inoculated (M), vector-infected (V), and $G m P A L$-silenced ( $\left.\mathrm{S}_{\mathrm{PAL}}\right)$ plants $\beta$-Tubulin levels were used as internal control for cDNA amounts. B, SA (left panel) and SA-glucoside (SAG, right panel) levels per gram fresh weight (FW) in $\mathrm{M}, \mathrm{V}$, and $\mathrm{S}_{\mathrm{PAL}}$ plants at 0 and $24 \mathrm{~h}$ postinoculation with virulent Pseudomonas syringae. C, Bacterial counts of virulent $P$. syringae in V (gray bars) or $\mathrm{S}_{\mathrm{PAL}}$ (black bars) plants. $\log _{10}$ values of CFU per unit leaf area from infected leaves at 0 and 4 days postinoculation (dpi) are presented. Error bars indicate standard deviation, $n=5$. Statistical significance was determined using Student's $t$ test. Asterisk denotes data significantly different from control (V) $(P<$ 0.001). D, Enzyme-linked immunosorbent assay of BPMV levels in the second and third trifoliolates of V (gray bars) or $\mathrm{S}_{\mathrm{PAL}}$ (black bars) plants at 14 dpi. Error bars indicate standard deviation, $n=4$. 
United Soybean Board (project 9244), USDA-NRI (2006-01854), and the Kentucky Soybean Promotion Board to A. Kachroo and S. Ghabrial.

\section{LITERATURE CITED}

Bilyeu, K. D., Palavalli, L., Sleper, D. A., and Beuselinck, P. R. 2003. Three microsomal omega-3 fatty-acid desaturase genes contribute to soybean linolenic acid levels. Crop Sci. 43:1833-1838.

Brooks, D. M., Bender, C. L., and Kunkel, B. N. 2005. The Pseudomonas syringae phytotoxin coronatine promotes virulence by overcoming salicylic acid-dependent defences in Arabidopsis thaliana. Mol. Plant Pathol. 6:629-639.

Browse, J. 2005. Jasmonate: an oxylipin signal with many roles in plants. Vitam. Horm. 72:431-456.

Browse, J. 2009. Jasmonate passes muster: a receptor and targets for the defense hormone. Annu. Rev. Plant Biol. 60:183-205.

Browse, J., and Howe, G. A. 2008. New weapons and a rapid response against insect attack. Plant Physiol. 146:832-838

Browse, J., McConn, M., James, D., Jr., and Miquel, M. 1993. Mutants of Arabidopsis deficient in the synthesis of alpha-linolenate. Biochemical and genetic characterization of the endoplasmic reticulum linoleoyl desaturase. J. Biol. Chem. 268:16345-16351.

Canakci, M., Monyem, A., and Van Gerpen, J. 1999. Accelerated oxidation processes in biodiesel. Trans. ASAE 42:1565-1572.

Chandra-Shekara, A. C., Gupte, M., Navarre, D. A., Raina, S., Raina, R., Klessig, D., and Kachroo, P. 2006. Light-dependent hypersensitive response and resistance signaling to turnip crinkle virus in Arabidopsis. Plant J. 45:320-335.

Chappell, A. S., and Bilyeu, K. D. 2006. A GmFAD3A mutation in the low linolenic acid mutant C1640. Plant Breed. 125:535-536.

Creelman, R. A., Tierney, M. L., and Mullet, J. E. 1992. Jasmonic $\mathrm{acid} /$ methyl jasmonate accumulate in wounded soybean hypocotyls and modulate wound gene expression. Proc. Natl. Acad. Sci. U.S.A. 89:4938-4941.

Cui, J., Bahrami, A. K., Pringle, E. G., Hernandez-Guzman, G., Bender, C. L., Pierce, N. E., and Ausubel, F. M. 2005. Pseudomonas syringae manipulates systemic plant defenses against pathogens and herbivores. Proc. Natl. Acad. Sci. U.S.A. 102:1791-1796.

Délano-Frier, J. P., Martínez-Gallardo, N. A., Martínez-de la Vega, O., Salas-Araiza, M. D., Barbosa-Jaramillo, E. R., Torres, A., Vargas, P., and Borodanenko, A. 2004. The effect of exogenous jasmonic acid on induced resistance and productivity in amaranth (Amaranthus hypochondriacus) is influenced by environmental conditions. J. Chem. Ecol. 30:1001-1034.

Fehr, W. R., Welke, G. A., Hammond, E. G., Duvick, D. N., and Cianzio, S. R. 1992. Inheritance of reduced linolenic acid content in soybean genotypes A16 and A17. Crop Sci. 32:903-906.

Flores, T., Karpova, O., Su, X., Zeng, P., Bilyeu, K., Sleper, D. A., Nguyen, H. T., and Zhang, Z. J. 2008. Silencing of GmFAD3 gene by siRNA leads to low alpha-linolenic acids (18:3) of fad3-mutant phenotype in soybean [Glycine max (Merr.)]. Transgenic Res. 17:839-850.

Fu, D.-Q., Ghabrial, S., and Kachroo, A. 2009. GmRAR1 and GmSGT1 are required for basal, $R$ gene-mediated and systemic acquired resistance in soybean. Mol. Plant-Microbe Interact. 22:86-95.

Fulton, J. P., Scott, H. A., and Gamez, R. 1980. Beetles. Pages 115-132 in: Vectors of Plant Pathogens. K F. Harris and K. Maramorosch, eds. Academic Press, New York.

Ghabrial, S. A., and Schultz, F. 1983. Serological detection of bean pod mottle virus in bean leaf beetles. Phytopathology 73:480-483.

Giesler, L. J., Ghabrial, S. A., Hunt, T. E., and Hill, J. H. 2002. Bean pod mottle virus: a threat to U.S. soybean production. Plant Dis. 86:12801289.

Hammond, E. G., and Fehr, W. R. 1983. Registration of A5 germplasm line of soybean. Crop Sci. 23:192.

Howe, G. A., and Jander, G. 2008. Plant immunity to insect herbivores. Annu. Rev. Plant Biol. 59:41-66.

Huang, S., Soldevila, A. I., Webb, B. A., and Ghabrial, S. A. 1997. Expression, assembly, and proteolytic processing of Helminthosporium victoriae 190S totivirus capsid protein in insect cells. Virology 234:130-137.

Kachroo, A., and Kachroo, P. 2007. Salicylic acid-, jasmonic acid- and ethylene-mediated regulation of plant defense signaling. Pages 55-83 in: Genetic Engineering, Principles, and Methods. J. Setlow, ed. Plenum Press, New York.

Kachroo, A., Fu, D.-Q., Havens, W., Navarre, D. A., Kachroo, P., and Ghabrial, S. A. 2008. An oleic acid-mediated pathway induces constitutive defense signaling and enhanced resistance to multiple pathogens in soybean. Mol. Plant-Microbe Interact. 21:564-575.

Kachroo, P., Yoshioka, K., Shah, J., Dooner, H. K., and Klessig, D. F. 2000. Resistance to turnip crinkle virus in Arabidopsis is regulated by two host genes and is salicylic acid dependent but NPR1, ethylene, and jasmonate independent. Plant Cell 12:677-690.

Kachroo, P., Shanklin, J., Shah, J., Whittle, E. J., and Klessig, D. F. 2001 A fatty acid desaturase modulates the activation of defense signaling pathways in plants. Proc. Natl. Acad. Sci. U.S.A. 98:9448-9453.

Kim, E. H., Kim, Y. S., Park, S. H., Koo, Y. J., Choi, Y. D., Chung, Y. Y., Lee, I. J., and Kim, J. K. 2009. Methyl jasmonate reduces grain yield by mediating stress signals to alter spikelet development in rice. Plant Physiol. 149:1751-1760.

Kloek, A. P., Verbsky, M. L., Sharma, S. B., Schoelz, J. E., Vogel, J., Klessig, D. F., and Kunkel, B. N. 2001. Resistance to Pseudomonas syringae conferred by an Arabidopsis thaliana COR-insensitive (coil) mutation occurs through two distinct mechanisms. Plant J. 26:509-522.

Lewsey, M. G., Murphy, A. M., MacLean, D., Dalchau, N., Westwood, J. H., Macaulay, K., Bennett, M. H., Moulin, M., Hanke, D. E., Powell, G., Smith, A. G., and Carr, J. P. 2010. Disruption of two defensive signaling pathways by a viral RNA silencing suppressor. Mol. PlantMicrobe Interact. 23:835-845.

Lomonossoff, G. P., and Ghabrial, S. A. 2001. Comoviruses. Pages 239242 in: Encyclopedia of Plant Pathology. O. C. Maloy and T. D. Murray, eds. John Wiley \& Sons, New York.

Love, A. J., Yun, B. W., Laval, V., Loake, G. J., and Milner, J. J. 2005. Cauliflower mosaic virus, a compatible pathogen of Arabidopsis, engages three distinct defense signaling pathways and activates rapid systemic generation of reactive oxygen species. Plant Physiol. 139:935948.

Mazarei, M., Elling, A. A., Maier, T. R., Puthoff, D. P., and Baum, T. J. 2007. GmEREBP1 is a transcription factor activating defense genes in soybean and Arabidopsis. Mol. Plant-Microbe Interact. 20:107-119.

Myhre, D. L., Pitre, H. N., Haridasan, M., and Hesketh, J. D. 1973. Effect of bean pod mottle virus on yield components and morphology of soybeans in relation to soil water regimes: a preliminary study. Plant Dis. Rep. 57:1050-1054.

Penninckx, I. A. M. A. , Thomma, B. P. H. J., Buchala, A., Métraux, J. P., and Broekaert, W. F. 1998. Concomitant activation of jasmonate and ethylene response pathways is required for induction of a plant defensin gene in Arabidopsis. Plant Cell 10:2103-2113.

Reddy, M. S. S., Ghabrial, S. A., Redmond, C. T., Dinkins, R. D., and Collins, G. B. 2001. Resistance to bean pod mottle virus in transgenic soybean lines expressing the capsid polyprotein. Virology 91:831-838.

Reinprecht, Y., Luk-Labey, S. Y., Larsen, J., Poysa, V. W., Yu, K., Rajcan, I., Ablett, G. R., and Pauls, K. P. 2009. Molecular basis of the low linolenic acid trait on soybean EMS mutant line RG10. Plant Breed. 128:253-258.

Ross, J. P. 1968. Effect of single and double infections of soybean mosaic and bean pod mottle viruses on soybean yield and seed characters. Plant Dis. Rep. 52:344-348.

Schwenk, F. W., and Nickell, C. D. 1980. Soybean green stem caused by bean pod mottle virus. Plant Dis. 64:863-865.

Selote, D., and Kachroo, A. 2010. RPG1-B derived resistance to AvrB expressing Pseudomonas syringae requires RIN4-like proteins in soybean. Plant Physiol. 153:1199-1211.

Skotland, C. B. 1958. Bean pod mottle virus of soybeans. Plant Dis. Rep. 42:1155-1156.

Ton, J., van Pelt, J. A., van Loon, L. C., and Pieterse, C. M. J. 2002. Differential effectiveness of salicylate-dependent and jasmonate/ethylenedependent induced resistance in Arabidopsis. Mol. Plant-Microbe Interact. 15:27-34.

Vlot, A. C., Dempsey, D. A., and Klessig, D. F. 2009. Salicylic acid, a multifaceted hormone to combat disease. Annu. Rev. Phytopathol. 47:177206.

Wilcox, J. R., and Cavins, J. F. 1985. Inheritance of low linolenic acid content of the seed oil of a mutant Glycine max. Theor. Appl. Genet. 71:74-78.

Yang, J. Y., Iwasaki, M., Machida, C., Machida, Y., Zhou, X., and Chua, N. H. 2008. BetaC1, the pathogenicity factor of TYLCCNV, interacts with AS1 to alter leaf development and suppress selective jasmonic acid responses. Genes Dev. 22:2564-2577.

Zhang, C., and Ghabrial, S. A. 2006. Development of Bean pod mottle virus-based vectors for stable protein expression and sequence-specific virus-induced gene silencing in soybean. Virology 344:401-411

Zhao, Y., Thilmony, R., Bender, C. L., Schaller, A., He, S. Y., and Howe, G. A. 2003. Virulence systems of Pseudomonas syringae pv. tomato promote bacterial speck disease in tomato by targeting the jasmonate signaling pathway. Plant J. 36:485-499.

\section{AUTHOR-RECOMMENDED INTERNET RESOURCE}

SoyBase and the Soybean Breeder's Tool website: www.soybase.org 